\title{
Habitat environmental factors influence intestinal microbial diversity of the short-faced moles (Scaptochirus moschata)
}

\author{
Lei Chen*, Di Xu, Jing Zhu, Shen Wang, Mi Liu, Mengyao Sun, Geyang Wang, Lingyu Song, Xiaoyu Liu and \\ Tianyu Xie
}

\begin{abstract}
The short-faced moles (Scaptochirus moschata) are unique Chinese mammal that live in burrows for life. They have complex ecological adaptation mechanisms to adapt to perennial underground life. Intestinal microbes play an important role in the ecological adaptation of wild animals. The gut microbiota diversity and its function in shortfaced moles' ecological adaptation is a scientific issue worth exploring. In this study, the Illumina HiSeq sequencing platform was used to sequence the V3-V4 hypervariable regions of the $16 \mathrm{~S}$ rRNA genes of 22 short-faced moles' intestinal samples to study the composition and functional structure of their intestinal microbiota. The results showed that in the short-faced moles' intestine, there are four main phyla, Firmicutes, Proteobacteria, Actinobacteria and Bacteroidete. At the family level, Peptostreptococcaceae and Enterobacteriaceae have the highest abundance. At the genus level, Romboutsia is the genus with the highest microbial abundance. According to the KEGG database, the main functions of short-faced mole gut microbes are metabolism, genetic information processing, environmental information processing, and cellular processes. The function of short-faced mole intestinal microbiota is suitable for its long-term burrowing life. No gender difference is found in the composition and function of the short-faced mole intestinal microbiota. There are significant differences in the composition and functional structure of the short-faced mole gut microbiota between samples collected from different habitats. We conferred that this is related to the different environment factors in which they live, especially to the edaphic factors.
\end{abstract}

Keywords: Short-faced moles, Gut microbiome, Gender, Sampling locations, Edaphic factor

\section{Introduction}

Short-faced mole (Scaptochirus moschata) belongs to the Mogera of the Talpidae, is a small mammal that lives in burrows for life, feeds on plant rhizomes and soil insects such as grubs, etc. It has developed morphological and physiological characteristics adapted to burrowing, such as eye degeneration, foreclaw valgus, smell and hearing sensitivity, etc. Short-faced mole is Chinese endemic species, mainly distributed in Henan, Shandong, Inner Mongolia, Ningxia, Shanxi and other provinces in China.

*Correspondence: leisurechen@163.com

College of Life Science, Qufu Normal University, Jingxuan West Street No.57, Qufu, Shandong Province 273165, China
Several studies on Talpidae have focused on the phylogenetic and morphological evolution, while the ecological adaptability of Talpidae is less studied.

Intestinal microbes are important symbionts in mammalian gut and occupy important niche (Willey et al. 2014; Saxena and Sharma 2016). They play an important role in maintaining intestinal health and immune function, including in the proliferation of intestinal cells, defense against pathogens, metabolism of secondary products, and digestion of complex compounds (Flint et al. 2012). Intestinal microbes can also affect animal nutrition metabolism and intestinal immunity through intestinal-brain axis (Schoster et al. 2017; Li et al. 2018; Wang et al. 2007), which is of great significance to animal 
survival and environmental adaptation (Wu et al. 2016; Zhu et al. 2011; Chen et al. 2017). There are many factors that affect gut microbes, including the genetic background of the host, the diet, age, gender, social class of the host, and the climate of the habitat. Soil, as an essential part of terrestrial mammal habitat, also plays an important role in the composition and diversity of animal gut microbes (Zhao et al. 2018; Wang et al. 2019).

Short-faced moles live in an underground hypoxic environment all year round. The physiological adaptation mechanisms are important and representative in the ecological research of burrowing mammals. The functional contribution of intestinal microbes in the burrowing life of the short-faced moles is an important field in the study of the physiological ecology of the burrowing mammals. Therefore, this study intends to study the gut microbial diversity of the short-faced moles, and to investigate the differences of intestinal microbes in moles with different sex and distribution. Furthermore, through the prediction of microbiome function, we also try to explore the function of gut microbes and their possible contribution in the adaptation to underground life of the short-faced moles. This study will provide data for the understanding of the physiological and ecological mechanism of shortfaced moles to adapt to burrowing life, and provide data for the ecological studies of small burrowing mammals.

\section{Materials and methods Samples collection}

In this study, 22 short-faced moles (SM) were collected using non-damagingly self-made mouse traps in September 2020, including 12 males (named M) and 10 females (named F). The sampling locations were Guanx$\operatorname{ian}\left(115^{\circ} \mathrm{E}, 36^{\circ} \mathrm{N}\right.$; named G; 11$)$ and Huimin $\left(117^{\circ} \mathrm{E}, 37^{\circ} \mathrm{N}\right.$; named $\mathrm{H} ; 11)$. The short-faced moles were captured and placed in the soil of the original habitat, and transported to the laboratory alive. Subsequently, the gasified diethyl ether was put into the transparent sealing device, and then the collected samples were put into the device, and the diethyl ether was continued. During this process, the state of short-faced moles was kept observing. After euthanasia, short-faced moles were dissected and the colon contents were collected aseptically. The collected colon contents samples were stored at $-80^{\circ} \mathrm{C}$ in the laboratory until DNA extraction.

This study was approved by the Medical Ethics Committee of Qufu Normal University (No. 2020018), and during the approval period, the short-faced moles was captured and dissected as required.

\section{DNA extraction, PCR amplification and sequencing} We used QIAamp DNA Stool Genomic DNA Extraction Kit (Qiagen, Germany) to extract DNA from intestinal samples according to the instructions. The concentration of the DNA was detected by NanoDrop ${ }^{\text {TM }}$ OneC (Thermo Fisher Scientifc, USA). We used qualified DNA samples for PCR amplification, then diluted the sample to $1 \mathrm{ng} /$ $\mu \mathrm{l}$ using sterile water. PCR amplification of the V3-V4 region of $16 \mathrm{~S}$ rRNA gene was performed with the primers 341F (CCTAYGGGRBGCASCAG) and 806R (GGA CTACNNGGGTATCTAAT). The PCR reaction steps were as follows: pre-denaturation at $98{ }^{\circ} \mathrm{C}$ for $1 \mathrm{~min}$; denaturation at $98^{\circ} \mathrm{C}$ for $10 \mathrm{~s}$, annealing at $55^{\circ} \mathrm{C}$ for $30 \mathrm{~s}$, extension at $72{ }^{\circ} \mathrm{C}$ for $30 \mathrm{~s}$ ( 35 cycles); and final extension at $72^{\circ} \mathrm{C}$ for $5 \mathrm{~min}$.

The concentration of PCR amplified products was detected by $2 \%$ agarose gel electrophoresis. Then the gel extraction kit (Qiagen, Germany) was used to purify the PCR products. The TruSeq ${ }^{\circledR}$ DNA PCR-Free Sample Preparation Kit was used to construct the library. The constructed library was quantitatively tested by Qubit. After the library was qualified, the Illumina HiSeq sequencing platform was used for sequencing.

\section{Sequencing data preprocessing}

According to the quality control process of Qiime (V1.9.1) (Caporaso et al. 2010), the raw tags obtained by sequencing were filtered strictly by quality control to obtain high-quality clean tags. Truncate Raw Tags from the first low-quality base site where the number of consecutive low-quality values (the default quality threshold is $<=19$ ) has reached the set length (the default length is 3). Then filter out the Tags whose continuous high-quality base length is less than $75 \%$ of the Tag length. After detect and remove chimeric sequences, the final effective tags were obtained for further analysis.

\section{OTUs classification and species abundance analysis}

To detect the species richness in each sample, the effective tags in all samples is classified as operational taxonomic units (OTUs) based on 97\% sequence similarity. Then the OTUs were compared to SILVA's SSUrRNA database for species annotation (set threshold of 0.8) by using Mothur software (Version 1.35.1). R software (v2.15.3) was used to generate Rarefaction curve and Rank Abundance curve. The end of the Rarefaction curve is gradually flattened, which proves the rationality of the sequencing amount, and the results obtained by sequencing can effectively reflect the abundance of microorganisms contained from the sample. We analyzed the OTUs distribution to study the species abundance of the gut microbes of each sample, and constructed a histogram of the relative abundance of OTUs at each classification levels (phylum, class, order, family, and genus). 


\section{Diversity analysis and significant test}

To analyze the diversity of the microbial community intragroup, we performed alpha diversity analysis by using Mothur software (Version 1.35.1) to reflect the abundance and diversity of the microbial community of each sample, including the observed-species (OTUs) abundance, chao1, Shannon, Simpson, and ACE indices (Li et al. 2013). Wilcoxon rank sum test was performed by using $\mathrm{R}$ software to evaluate the difference among the alpha diversity indices of different groups. $\mathrm{R}$ software was also used for beta diversity analysis based on Unweighted \& Weighted Unifrac distances to compare the microbial community composition intergroup. Multivariate statistical methods, include Principal Component Analysis (PCA), Principal Co-ordinates Analysis (PCoA) and Non-Metric Multi-Dimensional Scaling (NMDS) were performed to find the differences between different sample groups (Lozupone et al. 2005; Lozupone et al. 2011).

We used $\mathrm{R}$ vegan software package to perform BrayCurtis distance-based Anosim (Chapman et al. 1999), MRPP (O'Reilly et al. 1980) and ADONIS (Stat et al. 2013) analysis to assess whether the difference between groups was significantly higher than the difference within groups. Mothur software was used for AMOVA (Roewer et al. 1996) based on weighted and unweighted Unifrac distance to compare microbial diversity between different groups. LEfSe (Segata et al. 2011) was used to analyze the difference of abundance on each classification level (phyla, class, order, family, and genus) between groups, and to find the biomarkers that have a significant contribution to the difference between groups. Unweighted Pair-group Method with Arithmetic Means (UPGMA) trees based on Unweighted \& Weighted Unifrac distance were constructed to assess the similarity of the intestinal microbial diversity between different samples.

\section{Function prediction of short-faced moles intestinal microbiota}

We perform Tax4Fun function prediction by the Neighbor-Joining test based on minimum 16S rRNA gene sequence similarity. We extracted the $16 \mathrm{~S}$ rRNA gene sequence of the prokaryotic whole genome from the KEGG database and used the BLASTN algorithm to compare it to the SILVA SSU Ref NR database (BLAST bitscore $>1500$ ) to establish a correlation matrix. The KEGG database prokaryotic genome function information annotated by UProC and PAUDA methods was corresponding to the SILVA database to realize the function annotation of the SILVA database. Sequencing samples were clustered with the SILVA database sequence as the reference sequence OTUs, thus the functional annotation information was obtained (Aßhauer et al. 2015).

\section{Results}

Sequencing data processing and OTU analysis

A total of 2,085,117 raw paired-end reads are obtained from 22 short-faced moles intestinal samples. After quality control and chimera filtering, a total of 1,367,088 effective tags can be used for subsequent analysis with an average length of $411.91 \mathrm{bp}$. By clustered with $97 \%$ consistency, an average of 283 OTUs is obtained for each sample. According to the flower figure, there are only 17 OTUs shared by 22 samples, and the specificity between samples is relatively large (Fig. 1). The rarefaction curve and rank abundance curve indicate that sample size in this study is sufficient and can be used for subsequent analysis. Furthermore, the rarefaction curve (Additional file 1: Fig. S1) indicates that the intestinal microbial species abundance of SMGF06 is significantly higher than other samples. The rank abundance curve (Additional file 2: Fig. S2) also shows that the species abundance of SMGF06 samples is higher than that of other samples, but there is no obvious difference in species evenness.

\section{Gut microbiota composition of short-faced moles}

Except for unclassified microbial sequences and a little percentage of archaea, the short-faced moles intestinal microbiota includes 46 bacterial phyla, 60 classes, 138 orders, 270 families and 688 genera.

At the phylum level, Firmicutes (47.91\%) is the most abundant bacterial phylum, followed by Proteobacteria (31.36\%), Actinobacteria (13.43\%), Bacteroidete (3\%), Chloroflexi (0.97\%), Thaumarchaeota (0.55\%), Acidobacteri (0.525\%), Cyanobacteria (0.37\%) and Verrucomicrobia (0.32\%) (Fig. 2a). According to the sample collection area, we divided the samples into group Guanxian (SMG) and group Huimin (SMH). The bacterial phyla Candidatus_Jorgensenbacteria, Acetothermia, Gracilibacteria, Parcubacteria, Candidatus_Yanofskybacteria, Atribacteria, Candidatus_Nomurabacteri, Zixibacteria and Candidatus_Moranbacteria are only found in group SMG; phyla Candidatus_Kuenenbacteria, Berkelbacteria, Candidatus_Peregrinibacteria, Kiritimatiellaeota, Lentisphaerae and Candidatus_Woykebacteria are only found in group SMH (Additional file 3: Fig. S3). According to the gender of the samples, we divided the 22 samples into a male group (SMM) and a female group (SMF). The bacterial phyla of Candidatus_Kuenenbacteria, Crenarchaeota, Acetothermia, Berkelbacteria, Candidatus_Peregrinibacteria, Kiritimatiellaeota, Lentisphaerae and Candidatus_Woykebacteria are only found in group SMM; Parcubacteria, Hydrogenedentes, Candidatus_ Yanofskybacteria, Candidatus_Nomurabacteria, Zixibacteria, Candidatus_Moranbacteria are only found in group SMF (Additional file 4: Fig. S4). 


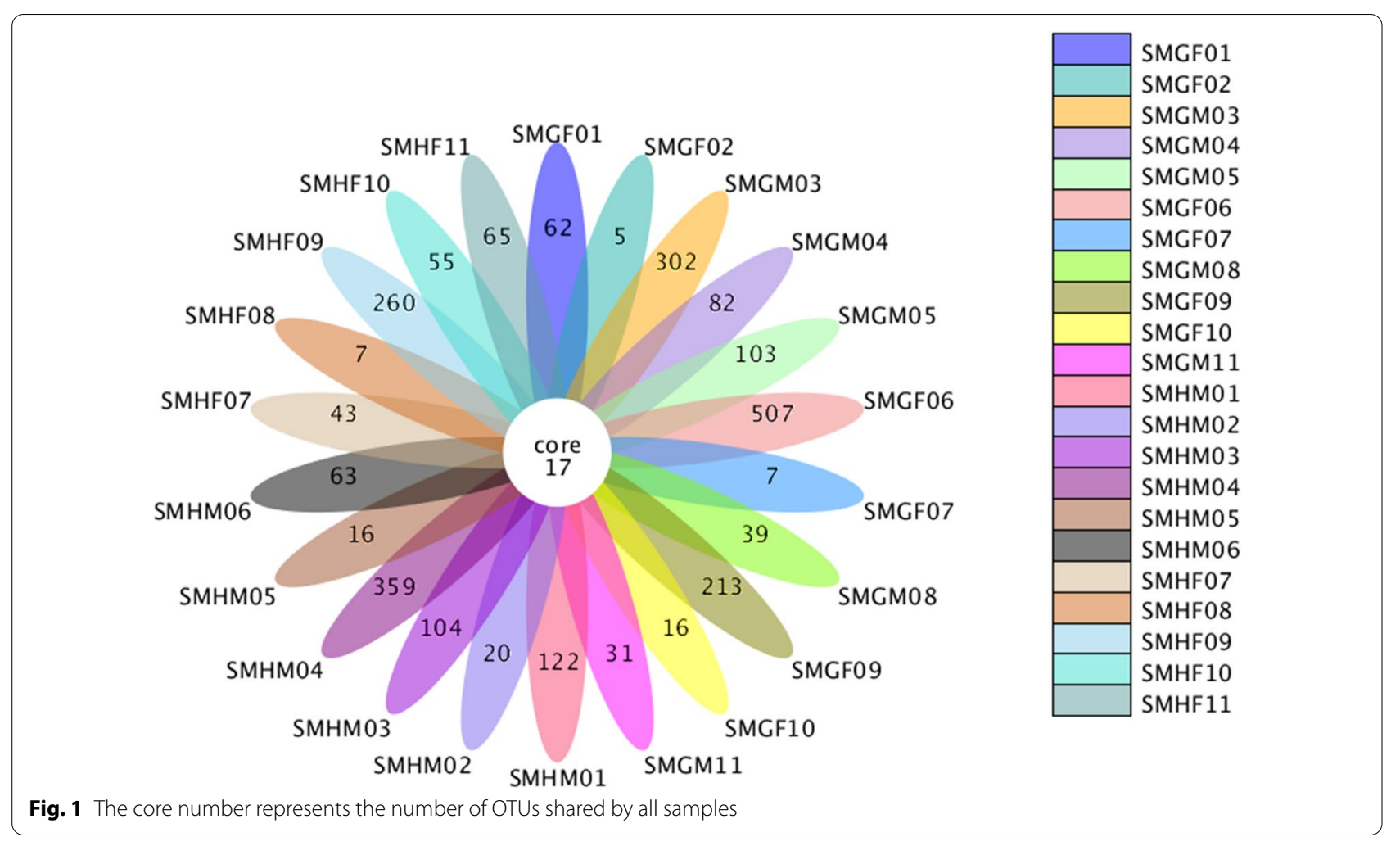

At the family level, the top ten abundant families are Peptostreptococcaceae (21.85\%), Enterobacteriaceae (15.95\%), Lachnospiraceae (6.63\%), Ruminococcaceae (3.56\%), Enterococcaceae (2.65\%), Bacillaceae (2.52\%), Christensenellaceae (2.30\%), Micrococcaceae (1.72\%), Streptomycetaceae (1.49\%) and Microbacteriaceae (1.23\%) (Fig. 2b). At the genus level, Romboutsia (15.27\%) is the most abundant genus, followed by nidentified Enterobacteriaceae (7.77\%), Enterobacter (6.34\%), Paeniclostridium (6.16\%), Shuttleworthia (2.37\%), Bacillus (2.27\%), Enterococcus (2.16\%), Streptomyces (1.49\%), Halomonas (0.89\%) (Fig. 2c).

\section{Comparison of intestinal microbial diversity between groups}

To test the similarity of microbial diversity in different samples from the same sampling location, we performed Anosim analysis based on the Bray-Curtis distance. The result showed that $\mathrm{SMH}$ vs SMG $\mathrm{R}=0.06965, \mathrm{P}=0.06$, which proved that the difference between groups was greater than the difference within groups (Additional file 5: Fig. S5). The Anosim analysis of the groups SMF and SMM showed that $\mathrm{R}=0.0009, \mathrm{P}=0.451$, proving that the difference between groups is also greater than the difference within the group (Additional file 6: Fig. S6). MRPP analysis and AMOVA analysis were also performed to further verify this result. The MRPP results showed that $\mathrm{SMG}$ vs $\mathrm{SMH} \mathrm{A}=0.01714, \mathrm{P}=0.036$, and SMF vs SMM $\mathrm{A}=0.0004643, \mathrm{P}=0.405$. The AMOVA analysis also showed that the difference both between the location groups and the gender groups was greater than that within the group, but the difference was all not significant (SMG vs SMH: weighted Unifrac AMOVA analysis, $\mathrm{P}=0.396$; unweighted, $\mathrm{P}=0.118$. SMF vs SMM: weighted Unifrac AMOVA analysis, $\mathrm{P}=0.306$; unweighted, $\mathrm{P}=0.31$ ).

We performed multiple regression analysis to explore whether there were significant differences in the gut microbes of short-faced moles between genders and between sample sites. The results showed that there was no significant difference between the groups SMH and SMG, and between the groups SMF and SMM (Table 1).

Based on the weighted and unweighted Unifrac distance, we conducted a beta diversity test between different sampling locations. Beta analysis based on weighted Unifrac distance showed no significant difference between the groups SMG and SMH $(\mathrm{P}=0.51)$, while beta diversity test based on the unweighted Unifrac distance showed extremely significant differences $(P=0.0033)$ (Fig. 3). Similarly, we also conducted a beta diversity test on the gut microbiota of different genders. The results showed that there was no significant difference between the groups SMM and SMF (weighted Unifrac beta analysis, $\mathrm{P}=0.7346$; unweighted Unifrac beta analysis, 

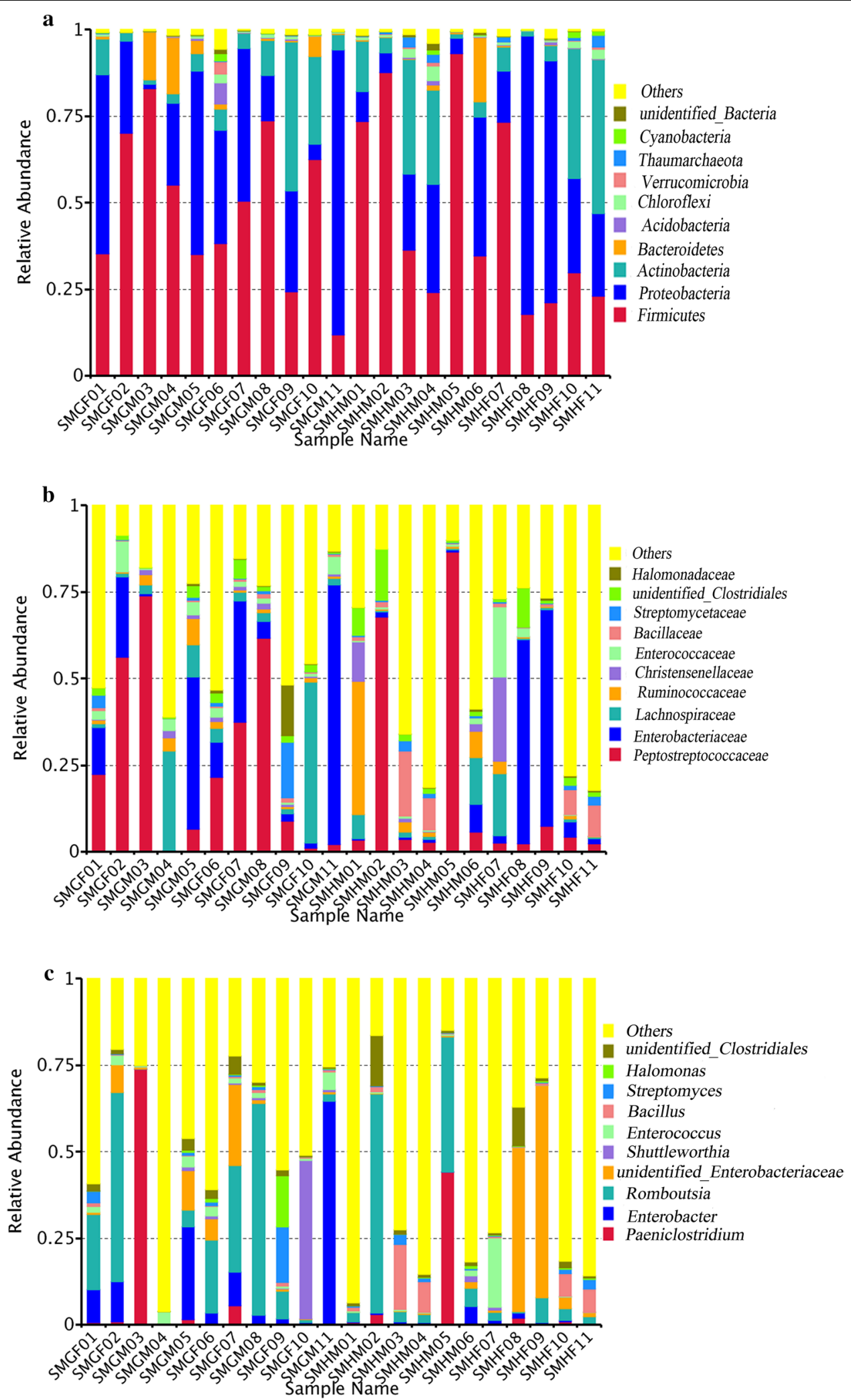

Fig. 2 Column chart of relative abundance of species at phylum (a), family (b), and genus (c) level 
Table 1 Multiple regression analysis of the influence of gender and sampling site on the alpha diversity of short-faced moles' intestinal microbial diversity

\begin{tabular}{lllllllr}
\hline Alpha index & OTUs & Shannon & Simpson & Chao1 & ACE & Goods coverage & PD whole tree \\
\hline Mean of SMF & 1103 & 5.140 & 0.846 & 1326.504 & 1380.497 & 0.993 & 114.570 \\
Mean of SMM & 1046 & 5.053 & 0.795 & 1326.272 & 1341.789 & 0.993 & 93.808 \\
Mean of SMH & 1155 & 5.617 & 0.850 & 1437.842 & 1447.486 & 0.993 & 102.052 \\
Mean of SMG & 993 & 4.576 & 0.791 & 1214.934 & 1274.800 & 0.993 & 106.326 \\
P value (SMF vs SMM) & 0.725 & 0.826 & 0.428 & 0.932 & 0.813 & 0.622 & 0.389 \\
P value (SMH vs SMG) & 0.413 & 0.214 & 0.379 & 0.356 & 0.447 & 0.805 & 0.919 \\
\hline
\end{tabular}

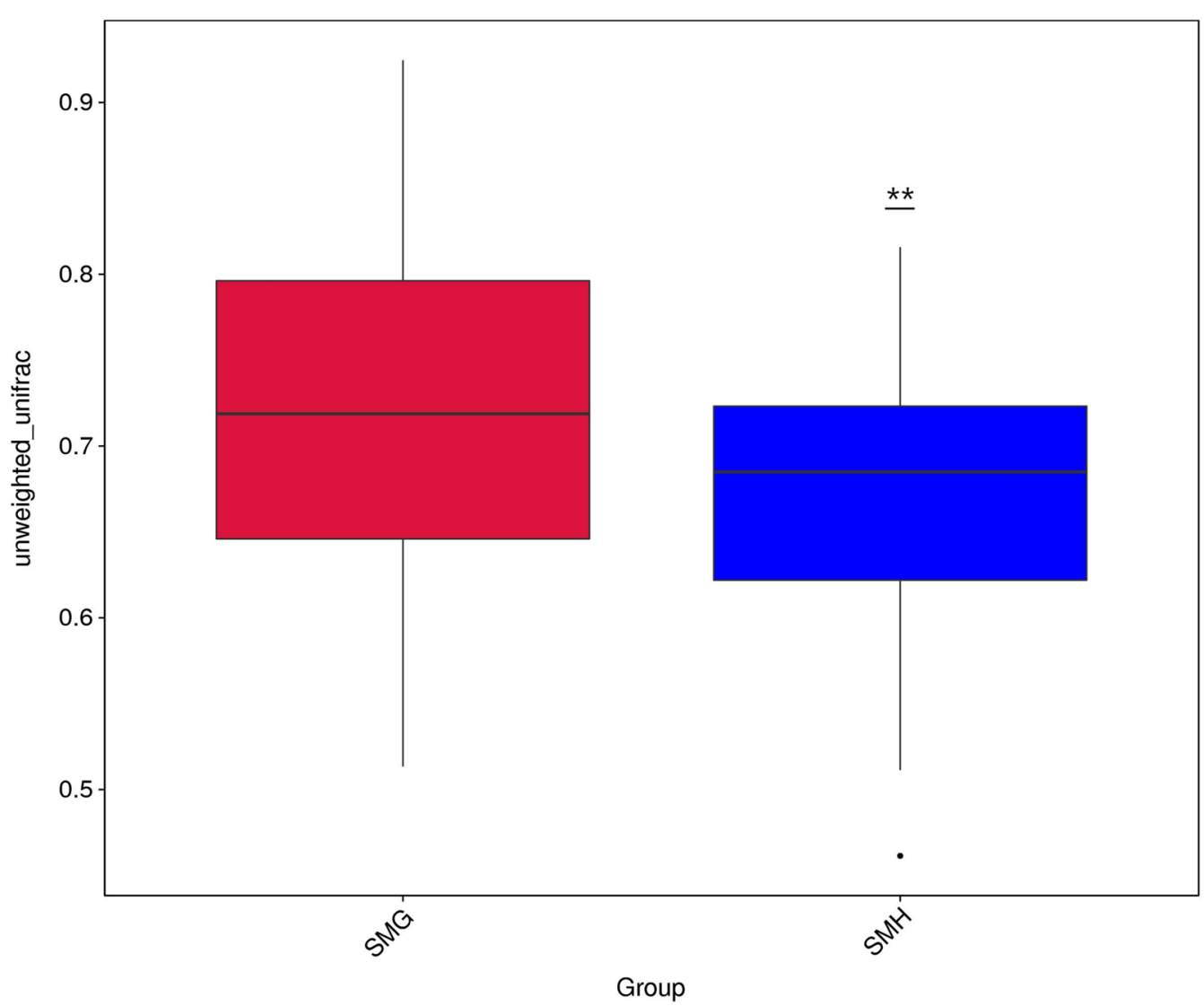

Fig. 3 Beta diversity test between different sampling locations based on unweighted unifrac distance

$\mathrm{P}=0.4176)$. In addition, by PCoA, PCA and NMDS analysis, we further analyzed the similarity between different samples. However, results of PCoA, PCA and NMDS analysis show that there is neither sampling-related aggregation nor gender-related aggregation (Additional file 7: Fig. S7, Additional file 8: Fig. S8, Additional file 9: Fig. S9, Additional file 10: Fig. S10, Additional file 11: Fig. S11 and Additional file 12: Fig. S12).

To further find out the differences between groups, we conducted wilcoxon tests between the groups on
OTU abundance at each classification level (phylum, class, order, family, genus). There are a total of 6 phyla, 1 class, 2 orders, 5 families, and 23 genera have significant differences between groups SMG and SMH, including the phyla Thaumarchaeota, Candidatus_Kuenenbacteria, Candidatus_Jorgensenbacteria, Berkelbacteria; the class Negativicutes; the orders Selenomonadales and Tepidisphaerale; the families Actinomycetaceae, Beutenbergiaceae, Paludibacteraceae, Actinomarinaceae, Parachlamydiaceae; and the genera Oxalobacter, 
Candidatus_Nitrososphaera, Rosenbergiella, Moryella, etc. There are 5 phyla, 2 classes, 1 order, 4 families, and 23 genera with significant differences between groups SMM and SMF, including the phyla Tenericutes, Candidatus_Kuenenbacteria, Crenarchaeota, Berkelbacteria, Acetothermia; the classes Thermoplasmata and Endomicrobia; the order Endomicrobiales; the families Paludibacteraceae, Endomicrobiaceae, Chromobacteriaceae, Parvularculaceae; and the genera Oxalobacter, Saccharofermentans, Moryella, Candidatus_ Nitrosotalea and Pirellula, etc. Then LEfSe analysis was performed to find the biomarkers that contribute most to the significant differences between the two groups. Results of LEfSe analysis shows that the biomarker that have significant differences between SMG group and SMH group are family Lachnospiraceae (SMG 9.25\%; SMH 4.01\%) and genus Enterobacter (SMG 11.76\%; SMH 0.91\%) (SMG $>$ SMH) (Fig. 4a). The biomarker between group SMM and group SMF are phylum Bacteroidetes; class Bacteroidia; family Ruminococcaceae (SMF 1.04\%; SMM 6.09\%) (SMF $<\mathrm{SMM);} \mathrm{order} \mathrm{Enterobacteriales,} \mathrm{family}$ Enterobacteriaceae (SMF 19.50\%; SMM 12.40\%); genus Unidentified-Enterobacteriaceae and Escherichia-coli (SMF > SMM) (Fig. 4b).

\section{Prediction of intestinal microbial function}

According to the function annotation information, the results show that the most important function of the intestinal microbiota of short-faced moles is metabolism ( 45.30\%), including carbohydrate metabolism ( 10.81\%), amino acid metabolism ( 9.14\%), energy metabolism $(\sim 4.45 \%)$, nucleotide metabolism $(\sim 3.86 \%)$, metabolism of cofactors and vitamins $(\sim 3.30 \%)$, lipid metabolism $(\sim 2.93 \%)$, etc. There is significant difference in the function abundance of carbohydrate metabolism and enzyme families between groups SMG and SMH

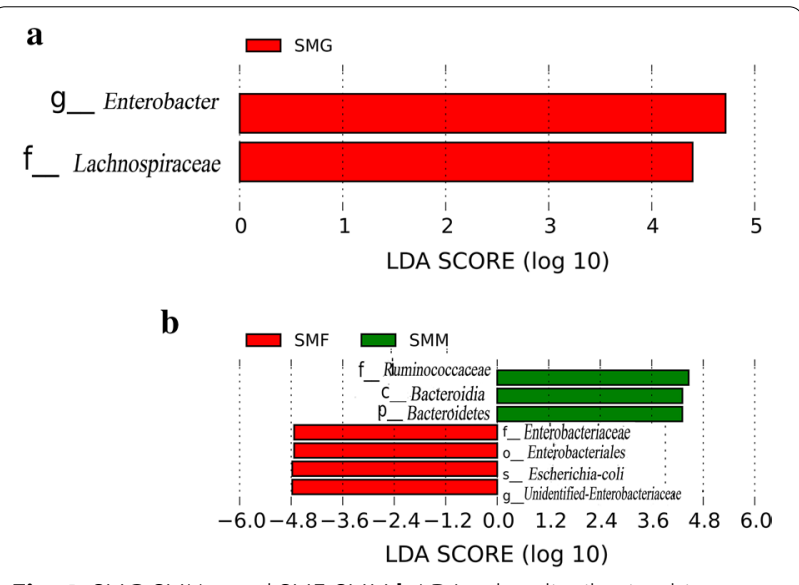

Fig. 4 SMG-SMH a and SMF-SMM $\mathbf{b} L D A$ value distribution histogram by Wilcoxon test $(\mathrm{P}<0.05)$. The second most important function is the genetic information processing ( 21.38\%), including translation $(\sim 9.59 \%)$, replication and repair $(\sim 8.44 \%)$, etc. There is significant difference in the function abundance of translation between the groups SMG and SMH $(\mathrm{P}<0.01)$. The other important function is environmental information processing $(\sim 14.38 \%)$, including membrane transport $(\sim 10.90 \%)$, signal transduction $(\sim 3.35 \%)$, etc. The function of intestinal microbes of short-faced moles is also associated with several diseases, such as infectious diseases, drug resistance and cancer, etc. Groups SMG and SMH have significant differences in the function process of cancer $(\mathrm{P}<0.05)$ (Fig. 5). At KEGG pathway level 3, the function of short-faced moles' intestinal microbiota is mainly annotated to transporters, two component system, DNA repair and recombination proteins, purine metabolism, transfer RNA biogenesis, $\mathrm{ABC}$ transporters, pyrimidine metabolism, amino acid related enzymes, quorum sensing, peptidases, etc. (Fig. 6, Additional file 13: Fig. S13).

\section{Discussion}

Short-faced mole is a small mammal that lives in underground caves all year round. Report on most aspects of short-faced mole remains scarce. In this study, we attempt to discuss the influence of gender and distribution area on the intestinal microbial diversity of the short-faced moles, and to find out whether there are significant differences in the gut microbes of short-faced moles of different genders or in different distribution locations. Through high-throughput sequencing of 22 short-faced moles' intestinal samples of different genders collected from two habitats, 46 phyla, 60 classes, 138 orders, 270 families and 688 genera are detected in the short-faced moles intestinal samples. At the phylum level, Firmicutes, Proteobacteria, Actinobacteria, and Bacteroidete are the dominant phyla. This is consistent with the results of other mammals (Xu et al. 2015; Zhang et al. 2010; Chen et al. 2016; Han et al. 2019; Ley et al. 2008). Among them, the phylum Firmicutes occupies $47.91 \%$ of the total intestinal microbiota, and its abundance fluctuates greatly between different samples (11.86\%-93.15\%). Some rodents, such as mice (Mus), have about $80 \%$ Firmicutes in their intestinal microbiota, and rat (Rattus norvegicu) contain Firmicutes at about $78.77 \%$ (Li et al. 2017). Among the herbivorous animals, such as bharals (Pseudois nayaur) (Chi et al. 2019), Tibetan wild ass (Equus kiang) (Gao et al. 2019), Firmicutes is also the dominant phyla of their gut microbes. Short-faced moles mainly feed on plant rhizomes, insect larvae, etc. The high abundance of Firmicutes can help short-faced moles enhance the digestion and absorption of plant cellulose. 


\section{Cellular Processes Cellular community - prokaryotes Cell growth and death Cell motility}

\section{Environmental Information Processinc Signal transduction Membrane transport}

\section{Genetic Information Processing}

Transcription

Replication and repair

Folding, sorting and degradation

\section{Human Diseases}

Substance dependence

Neurodegenerative diseases

Infectious diseases

Immune diseases

Endocrine and metabolic diseases

Drug resistance

Cardiovascular diseases

Cancers

\section{Metabolism}

Xenobiotics biodegradation and metabolism Nucleotide metabolism

Metabolism of terpenoids and polyketides

Metabolism of other amino acids

Metabolism of cofactors and vitamins

Lipid metabolism

Glycan biosynthesis and metabolism

Enzyme families

Energy metabolism

Carbohydrate metabolism

Biosynthesis of other secondary metabolites

Amino acid metabolism

\section{Organismal Systems}

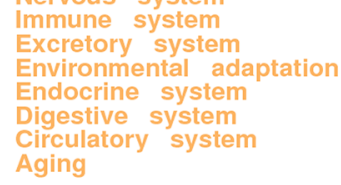

Immune system

Excretory system

Environmental adaptation

Endocrine system

Digestive system

Circulatory systen

Aging

\section{Unclassified}

\section{protein family}

Poorly characterized

Metabolism

Genetic information processing

Cellular processes and signaling

\section{KEGG pathway annotation}

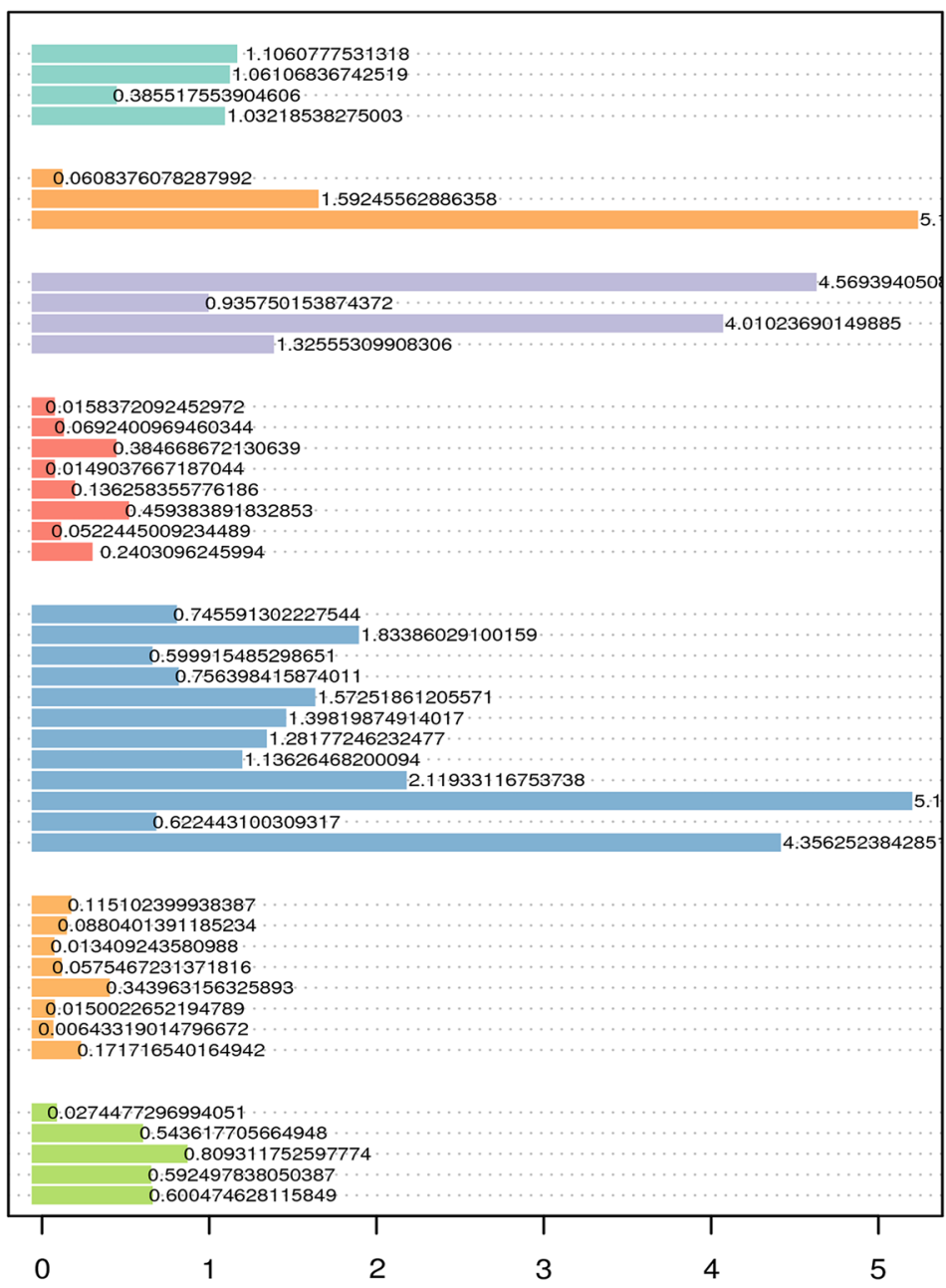

Reletive abundance of Gene

Fig. 5 KEGG pathway annotation of short-faced moles gut microbiota

Proteobacteria occupies $31.36 \%(1.19 \%-82.22 \%)$ of short-faced moles intestinal microbiota. The abundance of this phylum also varies greatly among individuals (82.22\% in sample SMGM11, but only $1.19 \%$ in sample SMGM03). Previous studies have shown that Proteobacteria is related to malnutrition of hosts suffering from metabolic or inflammatory diseases, and may be related to the dynamic establishment of the gastrointestinal microbiota of young animals (Moon et al. 2018). However, the samples we collected did not show gastrointestinal diseases. Whether Proteobacteria are related to short-faced moles' intestinal immunity needs further research.
Actinobacteria and Bacteroidete are also dominant phyla in the short-faced moles intestinal microbiota. Relevant studies have shown that an increase in protein and fat in animal foods will increase Actinobacteria abundance in intestinal microbiota (Guan et al. 2016). And carnivorous animals have high abundance of Actinobacteria in their gut may be associated with high fat and high protein feeding habits. In the present study, there is a high abundance of Actinobacteria in shortfaced moles intestinal microbiota (second to Proteobacteria). We speculate that the diet of the short-faced 

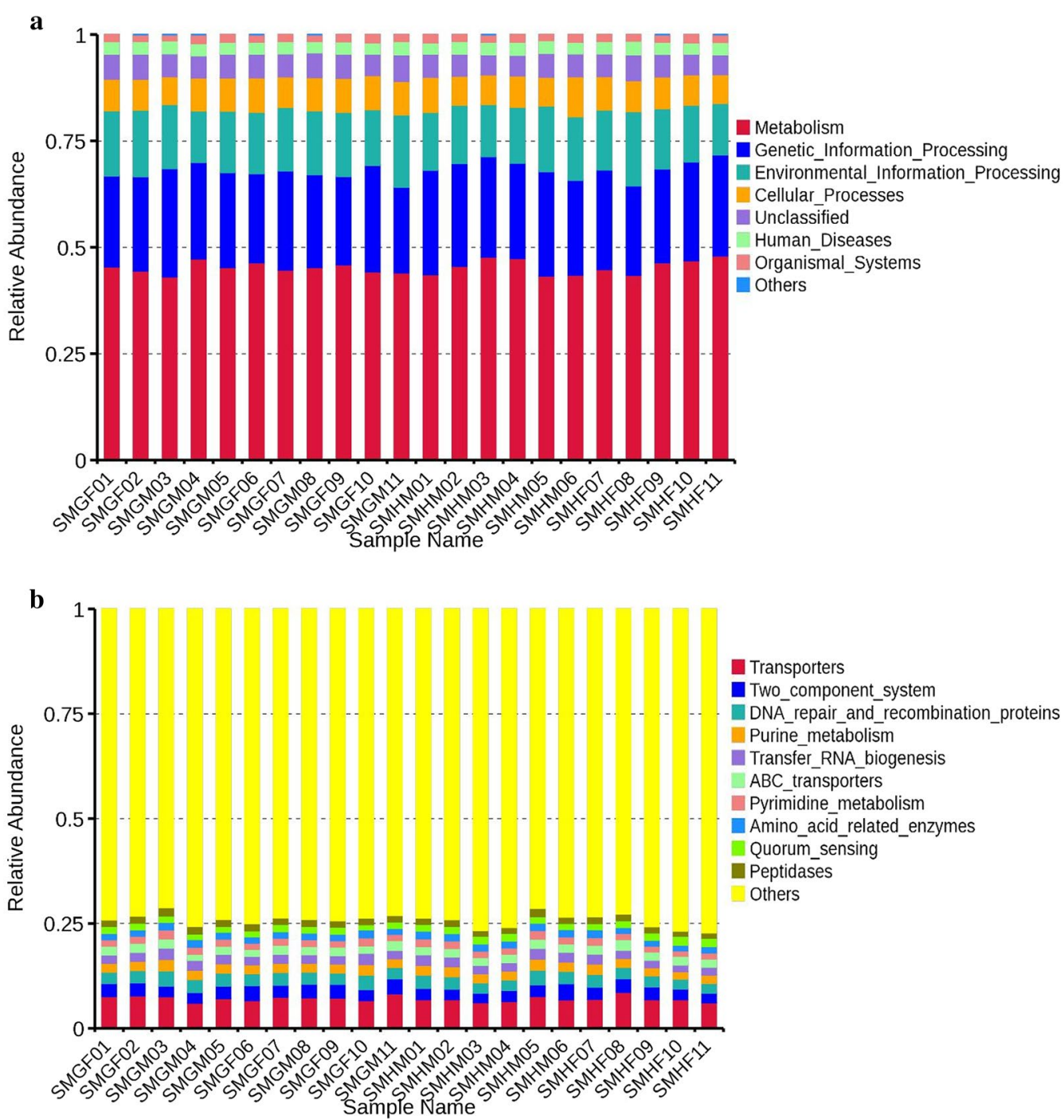

Fig. 6 Tax4Fun Function annotation histogram of relative abundance of level 1 (a) and level 3 (b)

moles may have a higher proportion of protein foods. Microbes of Bacteroidetes can promote digestion. They are the main bacteria that digest dietary polysaccharides and break down proteins. Bacteroidetes can help the host degrade high molecular weight organic matter, help the host degrade plant cell wall compounds (including cellulose, pectin and xylan), and play an important role in maintaining host intestinal health (Wu et al. 2011, 2016; Chen et al. 2017; Becker et al. 2014; Salyers et al. 1988). Compared with other mammals, such as bactrian camel (Camelus bactrianus)(20\%) (Wang et al. 2018a, b); rat (Rattus norvegicu) (9.12\%) (Li et al. 2017), the content of Bacteroidetes in the short-faced moles' intestinal microbiota is low (only $3.00 \%$ ), which is similar to the results of the study on the intestinal microbes of grey geese (Anser anser) (Wang et al. 2018a, b). According to the researchers, a low Bacteroidetes abundance of grey geese is due to dietary reasons, or other gut microbiota perform biopolymer degradation functions (Wang et al. 2018a, b). Therefore, we speculate that the low content Bacteroidetes in short-faced moles' intestinal microbiota may be related to their food composition. And we speculate that the dietary polysaccharides in the short-faced moles' diet take only a small percentage.

The beta diversity test results based on unweighted Unifrac distance showed that there are extremely significant differences between groups from different sampling locations. However, the beta diversity test results based on the weighted Unifrac distance showed no significant difference between the two groups. Study 
on the intestinal microbial community of free-range and captive cheetahs (Acinonyx jubatus) found that based on the weighted Unifrac distance analysis results have higher resolution than the results based on the unweighted Unifrac distance. Then they indicated that the difference between captive and free-range cheetahs is attributed more to the different abundance of several microbes than to the existence of the unique OTUs (Wasimuddin et al. 2017). We consider that the differences between different regional groups may also be caused by the differences in the abundance of some OTUs.

Previous studies have found that the composition and diversity of Arctic sediment is similar to that of local mammalian intestinal microbial flora (Wang et al. 2019). Researchers also found that the living environment of baboons (Papio), especially the geological history of soil and the content of exchangeable sodium, significantly affect the intestinal microbial flora (Grieneisen et al. 2019). In the present study, one of the sampling site (Huimin) is mainly a plain formed by sediment deposition of the Yellow River, and it is about 1,000 m away from the Yellow River. The other sampling site (Guanxian) is the alluvial plain of the Yellow River. Because of the diversion of the ancient Yellow River, it is more than $8000 \mathrm{~m}$ away from the Yellow River channel. Though examining the physical and chemical properties of the soil in the mole burrows of two sampling sites, we found that the amount of organic matter and cation exchange in soil of Huimin is lower than that in Guanxian. There are significant differences in cation exchange capacity $(\mathrm{cmol} /$ $\mathrm{kg})$, exchangeable sodium $\left(\mathrm{cmol}\left(\mathrm{Na}^{+}\right) / \mathrm{kg}\right)$ and organic matter $(\mathrm{g} / \mathrm{kg})(\mathrm{SMG}>\mathrm{SMH})$ in the soil between Huimin and Guanxian (Additional file 14: Table S1). Short-faced moles live in underground caves for a life time and get food from the soil. According to our testing, there are some significant differences in the soil chemistry between the two regions (Additional file 14: Table S1). Therefore, we speculate that soil factors will have a certain impact on the intestinal microbes of short-faced moles, but its influence mechanism is still unclear and needs further study.

UPGMA analysis found that the gut microbiota did not cluster associated with sampling locations. There are still large differences in gut microbes between different individuals in the same sampling location, which may be related to the difference in the microenvironment between individuals. Therefore, environmental factors in the microenvironment may have a more significant impact on the gut microbes of short-faced moles, which have a long-term burrowing underground life with poor migration ability. In addition, due to the number of samples from the same sampling location is still small, whether the microenvironment has more influence on the diversity of intestinal microbiota or the distribution area has more influence on intestinal microbiota steel needs further study.

Many studies have reported that intestinal microbial diversity in humans and many wild mammals is related to gender. Studies on the intestinal microbes of dholes (Cuon alpinus) found that the abundance of Bacteroidetes in the gut microbes of female samples was higher than that of males (Wu et al. 2016). Studies on golden takin (Budorcas taxicolor bedfordi) also found that the gut microbial diversity was affected by gender (Chen et al. 2017). In the present study, the results showed that there was no significant difference in the diversity and abundance of intestinal microbial communities in different gender groups by alpha diversity and beta diversity analysis. However, when compared the abundance of shortfaced moles intestinal microbial abundance of different gender groups at different classification levels, we found that there were significant differences between male and female groups in 5 phyla, 2 classes, 1 order, 4 families and 23 genera. By LEfSe analysis, we found the biomarkers of significant difference between group SMM and group SMF, included family Ruminococcaceae (SMF $<\mathrm{SMM}$ ) and family Enterobacteriaceae (SMF > SMM).

In this study, we find that the main functions of the intestinal microbiome of short-faced moles are metabolism, genetic information processing and environmental information processing, etc. By metagenome sequencing and function annotation of three wild giant panda intestinal samples, researchers identified homologous sequences with genomic genes encoding cellulase, glucosidase, xylanase, and xylanase. They believe that giant panda intestinal microbes play an important role in the metabolism of lactic acid, amino acids, xenobiotics, nucleotides, polysaccharides, vitamins and lipids in giant pandas (Zhu et al. 2011). A study on the Siberian tiger (Panthera tigris) suggests that the intestinal microbial gene function of the Siberian tiger is mainly related to carbohydrate metabolism subsystem and protein metabolism, respectively. Compared with breast-fed Siberian tiger cubs, the metabolism, translation, replication and repair of carbohydrates, amino acid metabolism, membrane transport and cofactor and vitamin metabolism in the intestinal microbial community of goat breast-fed Siberian tiger cubs decreased (He et al. 2018). Study on the intestinal microbial function of other small mammals, such as shrews (Tupaia belangeri), it is also found that most of the gene function of intestinal microbes are related to metabolism, and are more involved in amino acid metabolism and carbohydrate metabolism. We find 
that sugar metabolism (10.81\%), amino acid metabolism (9.14\%), energy metabolism (4.45\%) and nucleotide metabolism (3.86\%) account for a large proportion of the main metabolic function of short-faced moles intestinal microbiota. We speculate that this may because that, on one hand, it is beneficial to enhance the digestion and absorption ability of plant cellulose, and on the other hand, it can provide sufficiently energy to adapt to the low oxygen and lightless living environment, and meet the nutritional and energy needs of reproduction.

At the second level of KEGG pathway, the annotated gene function of short-faced moles' intestinal microbiota includes membrane transport, protein translation and other functions besides sugar metabolism, amino acid metabolism and other basic metabolisms. Some studies have shown that compared with captive cheetahs (Acinonyx jubatus), the intestinal flora functions of wild cheetahs mainly focus on environmental adaptation, membrane transport, immune system and translation. In order to adapt to environmental requirements such as food supply, competition, territory and family scope, wild cheetahs have enhanced these functions of intestinal flora. A study also found that immune-related genes are enriched in gut microbes in camels (Camelus bactrianus) about two years old (He et al. 2019). The gene function of short-faced moles intestinal microflora annotation shows that intestinal microbes also play an important role in the nutrient transport and metabolism of the short-faced moles, which may also be suitable for the life habits of long-term excavation.

In summary, the present study uses high-throughput sequencing technology to study the abundance, diversity and function of short-faced moles gut microbes, and the differences between gender groups and sampling location groups, which will provide data for the study of nutritional ecology of short-faced moles and other small burrowing mammals. In addition to sampling location and gender, other factors may also affect the diversity and function of mammalian gut microbiota. In later studies, the intestinal microbial diversity will be further associated with a variety of ecological factors, and the interaction between the diversity of intestinal microbes and environmental factors will be further studied. The sample size will be increased to draw more reliable conclusions. From the perspective of the mutual beneficial symbiosis of gut microbes and their hosts, it will open a new window to clarify the ecological adaptation mechanism of lifelong burrowing mammals.

\section{Supplementary Information}

The online version contains supplementary material available at https://doi. org/10.1186/s13568-021-01252-2.
Additional file 1:Figure S1. The rarefaction curve.

Additional file 2: Fig. S2. Rank abundance curve.

Additional file 3: Fig. S3. Composition of gut microbes at the phylum level in different sampling sites.

Additional file 4: Fig. S4. Intestinal microbial composition of different genders at the phylum level.

Additional file 5: Fig. S5. Analysis of differences between Anosim groups of different sampling locations.

Additional file 6: Fig. S6. Analysis of differences between Anosim groups of different genders.

Additional file 7: Fig. S7.PCoA analysis between groups of different sampling locations.

Additional file 8: Fig. S8. PCoA analysis between groups of different genders.

Additional file 9: Fig. S9.PCA analysis between groups of different sampling locations

Additional file 10: Fig. S10.PCA analysis between groups of different genders.

Additional file 11: Fig. S11.NMDS analysis between groups of different sampling locations.

Additional file 12: Fig. S12.NMDS analysis between groups of different genders.

Additional file 13: Fig. S13. Tax4Fun Function annotation histogram of relative abundance of level 2.

Additional file 14: Table S1. Soil physical and chemical properties in two regions (Mean $\pm \mathrm{SD}$ ).

\section{Acknowledgements}

Our thanks to Jiaxin Chen for her help in sample collection, and to the staff of Beijing Novogene Co. Ltd for their help in high-throughput sequencing and data analysis.

\section{Authors' contributions}

$L C$ conceived, designed, performed the experiments, ML analyzed the data; DX wrote the paper; JZ, SW, MS contributed materials; SW, GW, XL, TX, LS modify the manuscript. All authors read and approved the final manuscript.

\section{Funding}

This study is supported by the National Natural Science Foundation of China (31400473), and the Science and Technology Plan Project for Colleges and

Universities in Shandong Province of China (J14LE16).

\section{Availability of data and materials}

The raw data of the short-faced moles' intestinal microbiota $16 \mathrm{~S}$ rRNA genes could be found in the NCBI SRA database (PRJNA613028).

\section{Declarations}

\section{Ethics approval and consent to participate}

The capture and dissection of animals and the collection of intestinal samples involved in this study were approved by the Medical Ethics Committee of Qufu Normal University, and all procedures were carried out in accordance with the approval plan of the Medical Ethics Committee of Qufu Normal University (Approval number 2020018).

Consent for publication

The written informed consent forms were taken from the volunteers.

Competing interests

The authors declare that they have no conflict of interest. 
Received: 29 April 2021 Accepted: 15 June 2021

Published online: 23 June 2021

\section{References}

Aßhauer KP, Wemheuer B, Daniel R, Meinicke P (2015) Tax4Fun: predicting functional profiles from metagenomic 165 rRNA data. Bioinformatics 31(17):2882-2884

Becker AA, Hesta M, Hollants J, Janssens GP, Huys G (2014) Phylogenetic analysis of faecal microbiota from captive cheetahs reveals underrepresentation of Bacteroidetes and Bifidobacteriaceae. BMC Microbiol 14:43

Caporaso JG, Kuczynski J, Stombaugh J, Bittinger K, Bushman FD, Costello EK, Fierer N, Peña AG, Goodrich JK, Gordon Jl, Huttley GA, Kelley ST, Knights D, Koenig JE, Ley RE, Lozupone CA, McDonald D, Muegge BD, Pirrung M, Reeder J, Sevinsky JR, Turnbaugh PJ, Walters WA, Widmann J, Yatsunenko T, Zaneveld J, Knight R (2010) QIIME allows analysis of high-throughput community sequencing data. Nat Methods 7(5):335-336

Chapman MG, Underwood AJ (1999) Ecological patterns in multivariate assemblages: information and interpretation of negative values in ANOSIM tests. Marine Ecol Progr 180(3):257-265

Chen L, Zhang H, Liu G, Sha W (2016) First report on the bacterial diversity in the distal gut of dholes (Cuon alpinus) by using $16 \mathrm{~S}$ rRNA gene sequences analysis. J Appl Genet 57(2):275-283

Chen J, Zhang H, Wu X, Shang S, Yan J, Chen Y, Zhang H, Tang X (2017) Characterization of the gut microbiota in the golden takin (Budorcas taxicolor bedfordi). AMB Express 7(1):81

Chi X, Gao H, Wu G, Qin W, Song P, Wang L, Chen J, Cai Z, Zhang T (2019) Comparison of gut microbiota diversity between wild and captive bharals (Pseudois nayaur). BMC Vet Res 15(1):243

Flint HJ, Scott KP, Louis P, Duncan SH (2012) The role of the gut microbiota in nutrition and health. Nat Rev Gastroenterol Hepatol 9(10):577

Gao H, Chi X, Qin W, Wang L, Song P, Cai Z, Zhang J, Zhang T (2019) Comparison of the gut microbiota composition between the wild and captive Tibetan wild ass (Equus kiang). J Appl Microbiol 126(6):1869-1878

Grieneisen LE, Charpentier MJE, Alberts SC, Blekhman R, Bradburd G, Tung J, Archie EA (2019) Genes, geology and germs: gut microbiota across a primate hybrid zone are explained by site soil properties, not host species. Proc Biol Sci 286(1901):20190431

Guan Y, Zhang H, Gao X, Shang S, Wu X, Chen J, Zhang W, Zhang W, Jiang M, Zhang B, Chen P (2016) Comparison of the bacterial communities in feces from wild versus housed sables (Martes zibellina) by high-throughput sequence analysis of the bacterial 16S rRNA gene. AMB Express 6(1):98

Han S, Guan Y, Dou H, Yang H, Yao M, Ge J, Feng L (2019) Comparison of the intestinal microbiota of two free-ranging Chinese subspecies of the leopard (Panthera pardus) using high-throughput sequencing. Peer J. 28(7):e6684

He F, Liu D, Zhai J, Zhang L, Ma Y, Xu Y, Rong K, Ma J (2018) Metagenomic analysis revealed the effects of goat milk feeding and breast feeding on the gut microbiome of Amur tiger cubs. Biochem Biophys Res Commun 503(4):2590-2596

He J, Hai L, Orgoldol K, Yi L, Ming L, Guo F, Li G, Ji R (2019) High-throughput sequencing reveals the gut microbiome of the bactrian camel in different ages. Curr Microbiol 76(7):810-817

Ley RE, Hamady M, Lozupone C, Turnbaugh PJ, Ramey RR, Bircher JS, Schlegel ML, Tucker TA, Schrenzel MD, Knight R, Gordon JI (2008) Evolution of mammals and their gut microbes. Science 320(5883):1647-1651

Li B, Zhang X, Guo F, Wu W, Zhang T (2013) Characterization of Tetracycline resistant bacterial community in saline activated sludge using batch stress incubation with high-throughput sequencing analysis. Water Res 47(13):4207-4216

Li D, Chen H, Mao B, Yang Q, Zhao J, Gu Z, Zhang H, Chen YQ, Chen W (2017) Microbial biogeography and core microbiota of the rat digestive tract. Sci Rep 8:45840

Li Y, Hu X, Yang S, Zhou J, Qi L, Sun X, Fan M, Xu S, Cha M, Zhang M, Lin S, Liu S, Hu D (2018) Comparison between the intestinal bacterial microbiota of healthy and diarrheic captive musk deer. Front Microbiol 9:300

Lozupone C, Knight R (2005) Unifrac: a new phylogenetic method for comparing microbial communities. Appl Environ Microbiol 71(12):8228-8235

Lozupone CA, Hamady M, Kelley ST, Knight R (2007) Quantitative and qualitative beta diversity measures lead to different insights into factors that structure microbial communities. Appl Environ Microbiol 73(5):1576-1585
Lozupone C, Lladser ME, Knights D, Stombaugh J, Knight R (2011) Unifrac: an effective distance metric for microbial community comparison. ISME J 5(2):169-172

Moon CD, Young W, Maclean PH, Cookson AL, Bermingham EN (2018) Metagenomic insights into the roles of Proteobacteria in the gastrointestinal microbiomes of healthy dogs and cats. Microbiologyopen. 7(5):e00677

O'Reilly FJ, Mielkejr PW (1980) Asymptotic normality of MRPP statistics from invariance principles of U-statistics. Commun Stat 9(6):629-637

Roewer L, Kayser M, Dieltjes P, Nagy M, Bakker E, Krawczak M, de Knijff P (1996) Analysis of molecular variance (AMOVA) of Y-chromosome-specific microsatellites in two closely related human populations. Hum Mol Genet 5(7):1029-1033

Salyers AA, Pajeau M, Mccarthy RE (1988) Importance of mucopolysaccharides as substrates for Bacteroides thetaiotaomicron growing in intestinal tracts of exgermfree mice. Appl Environ Microbiol 54(8):1970-1976

Saxena R, Sharma VK (2016) A metagenomic insight into the Human microbiome: its implications in health and disease. Med Health Genomics 107-119

Schoster A, Staempfli HR, Guardabassi LG, Jalali M, Weese JS (2017) Comparison of the intestinal bacterial microbiota of healthy and diarrheic foals at two and four weeks of life. BMC Vet Res 13(1):144

Segata N, Izard J, Waldron L, Gevers D, Miropolsky L, Garrett WS, Huttenhower C (2011) Metagenomic biomarker discovery and explanation. Genome Biol 12(6):R60

Stat M, Pochon X, Franklin EC (2013) The distribution of the thermally tolerant symbiont lineage (Symbiodinium, clade D) in corals from Hawaii: correlations with host and the history of ocean thermal stress. Ecol Evol 3(5):1317-1329

Wang Q, Garrity GM, Tiedje JM, Cole JR (2007) Naive Bayesian classifier for rapid assignment of rRNA sequences into the new bacterial taxonomy. Appl Environ Microbiol 73(16):5261-5267

Wang Z, Zhang W, Wang B, Zhang F, Shao Y (2018a) Influence of bactrian camel milk on the gut microbiota. J Dairy Sci 101 (7):5758-5769

Wang W, Liu Y, Yang Y, Wang A, Sharshov K, Li Y, Cao M, Mao P, Li L (2018b) Comparative analyses of the gut microbiota among three different wild geese species in the genus Anser. J Basic Microbiol 58(6):543-553

Wang N, Guo Y, Li G, Xia Y, Ma M, Zang J, Ma Y, Yin X, Han W, Lv J, Cao H (2019) Geochemical-compositional-functional changes in Arctic soil microbiomes post land submergence revealed by metagenomics. Microbes Environ 34(2):180-190

Wasimuddin MS, Melzheimer J, Thalwitzer S, Heinrich S, Wachter B, Sommer S (2017) Gut microbiomes of free-ranging and captive Namibian cheetahs: Diversity, putative functions and occurrence of potential pathogens. Mol Ecol 26(20):5515-5527

Willey JM, Sherwood L, Woolverton CJ (2014) Prescott's Microbiology, 9th edn. McGraw-Hill, New York, NY, pp 713-721

Wu GD, Chen J, Hoffmann C, Bittinger K, Chen YY, Keilbaugh SA, Bewtra M, Di Knights H, Bushman FD, Lewis JD (2011) Linking long-term dietary patterns with gut microbial enterotypes. Science 334(6052):105-108

Wu X, Zhang H, Chen J, Shang S, Wei Q, Yan J, Tu X (2016) Comparison of the intestinal microbiota of dholes high-throughput Illumina sequencing of the $\mathrm{V} 3-\mathrm{V} 4$ region of the $16 \mathrm{~S}$ rRNA gene. Appl Microbiol Biotechnol 100(8):3577-3586

Xu B, Xu W, Li J, Dai L, Xiong C, Tang X, Yang Y, Mu Y, Zhou J, Ding J, Wu Q, Huang Z (2015) Metagenomic analysis of the Rhinopithecus Bieti intestinal microbiome reveals a broad diversity of bacterial and glycoside hydrolase profiles related to lignocellulose degradation. BMC Genomics 16(1):174

Zhang H, Chen L (2010) Phylogenetic analysis of 165 rRNA gene sequences reveals distal gut bacterial diversity in wild wolves (Canis lupus). Mol Biol Rep 37(8):4013-4022

Zhao J, Yao Y, Li D, Xu H, Wu J, Wen A, Xie M, Ni Q, Zhang M, Peng G, Xu H (2018) Characterization of the gut microbiota in six geographical populations of Chinese Rhesus Macaques (Macaca mulatta), implying an adaptation to high-altitude environment. Microb Ecol 76(2):565-577

Zhu L, Wu Q, Dai J, Zhang S, Wei F (2011) Evidence of cellulose metabolism by the giant panda gut microbiome. Proc Natl Acad Sci USA 108(43):17714-17719

\section{Publisher's Note}

Springer Nature remains neutral with regard to jurisdictional claims in published maps and institutional affiliations. 